\title{
动态生态位: 构建群落生态学理论的新框架
}

\author{
牛克昌 ${ }^{1 *}$, 储诚进 $^{2}$, 王志恒 ${ }^{3}$ \\ 1. 南京大学生命科学学院, 南京 210023 ; \\ 2. 中山大学生态学院, 广州 510006; \\ 3. 北京大学生态研究中心, 北京大学城市与环境学院, 北京 100871 \\ *联系人, E-mail: kechangniu@nju.edu.cn
}

收稿日期: 2021-07-26; 接受日期: 2021-09-16; 网络版发表日期: 2022-01-04

国家自然科学基金(批准号:31870402)资助

\begin{abstract}
摘要 生态学研究的宗旨是澄清有机体与生物和非生物环境之间的关系. 作为生态学研究的基石, 生态位概念的 提出用以刻画物种的生态学意义, 量化其对环境的需求及影响, 从而揭示调控物种分布、群落构建、食物网动态 等生态学过程的机理. 生态位概念发展已有百年历史, 但人们对其内涵、外延等依然不甚了解, 不知如何量化, 生 态位相关理论面临严峻考验。为此, 本文简要追溯生态位概念发展的主要历程和脉络, 梳理生态位概念与竞争排 除、物种共存和群落构建等群落生态学核心理论之间的关系; 从生物与环境互馈演化的视角, 再思生态位何以能 解释几乎所有的生态学现象, 却又难预测任何机理和模式. 本文认为生态位不仅是物种生态学作用和影响的体 现、是群落动态的驱动因素, 更是有机体适应群落环境的结果; 是理解生态学规律的必要概念框架, 却难以成为 解析方法. 进而提出动态生态位 (dynamic niche) 概念框架, 以整合有机体属性变化、生态-演化互馈以及随机过程 对物种适合度动态和群落构建的重要影响, 更新生态位概念, 为重构群落生态学理论铺垫基础.
\end{abstract}

关键词生态位, 群落构建, 生物多样性, 物种共存, 中性理论

早在2500多年前中国古代先哲老子、庄子等就从 整体论的角度去解释生物与环境互馈影响为一整体, 古希腊哲学家赫拉克利特、亚里士多德等也强调从整 体自然中去深究局部万物的存在. 然而, 自 18 世纪牛顿 力学观盛行、19世纪达尔文用自然选择和性状演化解 释生物多样性起源和模式, 20 世纪的生物学家们甚至 认为还原论是揭示生命现象与自然规律的不二逻辑. 时至今日，许多生物学家们依然坚持澄清基因、分子 等的微观机理便可以揭示所有生命现象演变的规律. 在此背景下, 生态学从其诞生起, 就侧重于从个体、种
群和物种与环境的关系，揭示整体群落结构和功能动 态. 作为物种生态学意义的体现, 生态位(ecological niche)概念的提出和发展就致力于量化物种对周围环 境的需求及影响, 以期阐明调控物种的分布、共存和 群落构建等生态学过程的机理. 然而, 历经百年发展, 人们对生态位的内涵和外延依然不甚了解, 不知如何 量化. 生态位始终是一个玄之又玄的抽象概念, 被用 以解释几乎所有的生态学现象, 但又难预测任何机理 和模式.

21 世纪起，生态学家们开始质疑生态位是否可以

引用格式: 牛克昌, 储诚进, 王志恒. 动态生态位: 构建群落生态学理论的新框架. 中国科学: 生命科学, 2022, 52: 403-417

Niu K C, Chu C J, Wang Z H. Dynamic niche: a new foundation for rebuilding theory of community ecology (in Chinese). Sci Sin Vitae, 2022, 52: 403417, doi: $10.1360 /$ SSV-2021-0160 
量化, 生态位分化是否是物种共存的充分必要条件, 种 间生态位差异对群落构建是否起决定性作用; 甚至一 些学者开始探讨生态位本身的存在及演化、群落和个 体水平的生态位等一系列生态学根本问题 ${ }^{[1,2]}$. 另一方 面, 近十年来随着应用生态学兴起, 越来越多的研究侧 重于以大数据描述各种变化莫测的生态学现象及变量 相关性，使得生态位等传统生态学理论的检验和发展 成为研究的边缘主题 ${ }^{[3,4]}$,一些学者甚至呼吁彻底抛弃 一些生态位核心概念 ${ }^{[5]}$. 为此, 我们认为非常有必要追 溯生态位概念发展的主要历程和脉络, 梳理生态位理 论与竞争排除法则、物种共存和群落构建等群落生态 学核心理论之间的关系，从整体论思想以及生物与环 境互馈演化的视角, 结合随机过程的重要性, 再思生 态位是什么, 它为何难以成为揭示生态学规律的解析 方法，而其抽象思想又不可或缺. 我们深知在浩瀚的 文献中阐明生态位及其理论之难为，但又鲜见此类批 判性审视，特此勉力抛砖引玉，以期国内同行重视继 承和发扬传统生态学理论.

\section{1 生态位: 一个抽象的生态学基本概念}

1866年Haeckel定义的生态学(Ökologie)一词就指 研究(logía)居住地(oîkos)的学科, 居住地的主体指个 体、种群和物种, 甚至可扩展至群落有机体. 随之, 不 同物种分离而居的现象及原因就成为生态学家们关注 的核心主题. 1910年, Johnson ${ }^{[6]}$ 用生态位(niche, 可能源 自于法语Nicher，意为生态拿位nest)表征不同物种对 环境的不同需求; 1917年, Grinnell正式提出的生态位 概念更像是对生态学定义的具体化，他所定义的生态 位指物种或亚种在生境中存活所必需的一系列生物和 非生物环境条件 ${ }^{[7]}$, 强调物种存活对环境条件需求与 环境对物种影响的匹配关系 ${ }^{[8]}$. 实际上, 物种在所处环 境中有特定生境需求的思想，可追溯到18世纪中叶的 Liebig最小因子定律以及达尔文的自然选择. Grinnell 的定义系统地强调了生态位的有无相生及其内涵和外 延, 即 ( i ) 生物和非生物环境因子, 可划分为有明确地 理内涵的生物界(kingdoms)、区域(regions)、生物带 (life zones)等宏观高层次生态水平 ${ }^{[8]}$, 以及没有明确地 理内涵的生物与环境关系等低层次生态学研究对象 ${ }^{[9]}$; (ii) 作为物种存活和分布的环境终极单位(ultimate unit)，生态位可被看作是物种维持和繁殖后代所需的

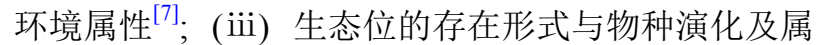
性有关，演化趋同使同种不同地区的种群有相似的生 态位, 即生态位是物种属性的生态学体现 ${ }^{[8]}$; (iv) 扩散 限制使一些可利用(环境)生态位可能未被物种占据, 形成生态位空缺 ${ }^{[8]}$. 值得提及的是生态位从正式定义 至今，其主体指“物种”，但在实际应用中却指特定地 区特定时间某物种的种群. 与此Grinnell的生态位不 同，1927年Elton ${ }^{[10]}$ 将生态位定义为有机体(物种)在食 物网(群落)中的地位和功能作用, 强调了物种在营养 级(群落环境)中的角色, 如某物种与其食物和捕食者 之间的关系. 他甚至认为, 动物生态位在一定程度上 可用其个体大小和食性量化; 同一物种在不同群落可 能有不同的生态位，同一生态位又被不同的物种所占 据 ${ }^{[10]}$.

此后，很多著名的生态学家都对生态位概念做了 不同程度的修订和扩展，但从根本上各种定义依然在 Grinnell和Elton的基本框架之内，只是侧重不同而 $己^{[11]}$. 例如, 1952年Dice ${ }^{[12]}$ 声称生态位本身不直接指 物种在群落中的功能, 而指物种对生境的占据和瓜分, 其生态功能是物种在环境中存活的结果. 1954年, Clarke ${ }^{[13]}$ 区分了侧重描述物种环境需求的生境生态位 (place niche, habitat niche)与物种影响环境的功能生态 位(functional niche), 在复杂的局域生态系统中, 不同 动植物物种功能不同; 但在不同的地理区域, 相同的功 能生态位可能被不同物种所占据. 简而言之, Grinnell 与Elton的生态位也没本质区别, 都指生物与环境的相 互作用，只是侧重不同(图1). 此后，侧重有机体(物种) 对环境需求的生境生态位, 被广泛应用于生物地理学 研究 ${ }^{[14,15]}$; 而侧重有机体(物种)影响其他生物及环境 的功能生态位, 在群落生态学研究中被发扬光大. 从 这个意义上，生态学几乎可以被定义为研究生态位的 科学 ${ }^{[16]}$. 值得一提的是无论何种释义, 同种或不同物 种在不同地理区域可能会有一致生态位(ecological equivalents)的思想在生态位早期定义中都被广泛提 及, 即生态位可能是物种适应环境属性的结果, 而不一 定是物种的固有属性 ${ }^{[17]}$. 但随着竞争排斥原理 (Competitive Exclusion Principle)强调生态位差异是物种共 存的必要条件, 生态位开始作为物种属性和原因变 量 $^{[16]}$.

1934年, Gause ${ }^{[18]}$ 首次以Lotka-Volterra种间竞争模 型中的竞争系数量化了Elton生态位，并以均匀资源且 


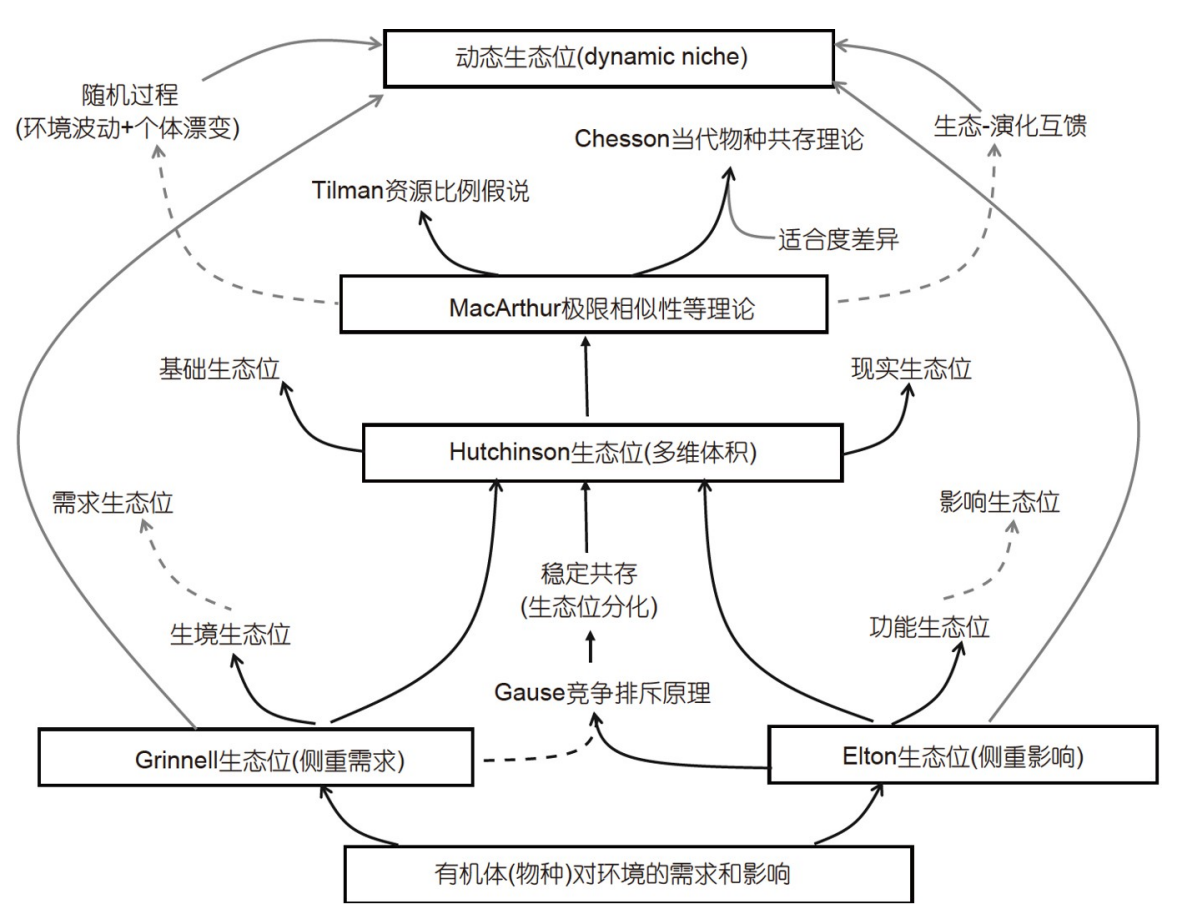

图 1 生态位主要概念及相关理论发展脉络示意图

Figure 1 Diagram of evolutionary linkage among key concepts of the ecological niche and relevant theories

有限空间下的双小核草履虫和大草履虫竞争实验，严 格证实了资源利用相同(需求生态位重叠)的两物种在 特定空间环境中难以稳定共存. 1948年, Park ${ }^{[19]}$ 也用面 粉甲壳虫实验得到了类似的结论. 虽然生态位相似的 物种难以稳定共存这种实验事实, 并不完全等价于物 种稳定共存必以生态位分化(niche differentiation)为前 提, 竞争排斥原理的直接逻辑后果是具有完全相同生 态位的物种不能稳定共存，生态位就被看作是种间确 定性作用的代名词. 至此, 竞争排斥原理成为生态位 及相关群落生态学理论发展的基石. 值得提及的是, 同期法国动物学家L'Héritier及Teissier ${ }^{[20]}$ 用果蝇做了 相似实验, 发现两个物种利用相同资源时, 能在一种近 似平衡的状态中长期共存. 此类明显违反竞争排斥原 理的研究此后很少被提及, 也从未挑战竞争排斥原理 在生态学中的神圣地位. 更重要的是, 实验条件下的 稳定共存与自然生态系统中的稳定共存并不等价. 实 际上据我们所知，在环境随机波动及个体扩散无处不 在的自然群落中, 难以证实竞争排斥原理, 也从未有 研究在自然系统中严格证实过. 但这似乎并未妨碍生 态学家量化各物种生态位及其差异程度, 将生态位概 念从抽象带至具体化, 使其逐步成为解释物种分布、
群落构建和动态的因变量, 形成生态位理论(图1).

\section{2 群落生态位理论：引领生态学从现象描 述向假设检验}

如果说生态位发展初期只是理解生物与环境关系 的抽象概念框架, Hutchinson对生态位的重新定义和 发展, 则将生态位概念具体化. 基于此的群落生态位 理论更使后来学者对生态学的认识发生了根本性变化 一生态学从现象描述转为假设检验式的科学.

\subsection{Hutchinson生态位概念及群落生态位理论}

1957年, Hutchinson ${ }^{[21]}$ 认为生态位是物种存活及 更新所需的一系列环境条件的集合，这个集合是总体 环境空间的子集, 其大小取决于物种适应性; 而物种 适应性又取决于长期演化形成的形态结构和生理特 征, 即物种属性决定其生态位; 因此每个物种有且只有 一个生态位, 也就没有生境中生态位空缺之说 ${ }^{[16]}$. 这 一概念不仅弱化了生态位初义, 甚至完全忽视了个体 行为适应及生物-环境反馈影响对生态位形成的作用, 即Odum ${ }^{[22]}$ 所强调的行为生态位(behavioral niche). 通 
过将生态位看作是与生存环境有关的物种属性, 生态 位概念不再抽象, 而是可被量化的生物属性; 与之相 互作用的非生物环境则被称为群落生境(biotope); 生 态位与群落生境相互对应, 形成二元对偶(niche-biotope duality ${ }^{[23]}$. 据此, 生态位可被量化为限制物种(在 某生态系统中)存活的所有环境条件和资源的多维体 积 ( $n$-dimensional hypervolume), 其潜在的假设是: ( i ) 生物所需的各种资源变量相互独立, 是多资源维度的 子集; (ii) 物种在各环境资源维度上的适合度密度(fitness density)呈正态分布, 在多维资源中就呈球形. 物 种最优适合度(optimal fitness)应出现于各环境资源最 优之处(即多维体积的中心), 离中心越远适合度越 低 $^{[21]}$. Hutchinson ${ }^{[21]}$ 进一步区分了无竞争等生物相互 作用影响下的基础生态位(fundamental niche), 以及自 然群落中竞争等种间互作影响下的现实生态位(realized niche). 具体地, 基础生态位指限制物种生存的多 维非生物环境超级体, 主要取决于物种属性依赖的生 物适应能力; 现实生态位指物种实际所能占具的多维 环境空间, 归根到底取决于物种在食物网及生命树中 的位置; 在生物地理学研究中也常包含扩散限制的影 响 $^{[16]}$. 理论上，区分和量化基础生态位和现实生态位 以及二者之间的差异, 就可以澄清物种对非生物环境 的需求及其对环境和其他生物的影响. 然而, 值得谨 记的是在一个真实的自然生态系统中, 无法孤立物种 与生物和非生物环境的互馈影响, 所能观测到的只是 物种的现实生态位.

根据对生态位的重新定义, Hutchinson及其跟随 者提出了生态位维度(niche dimensionality)、生态位 重叠(niche overlap)、生态位分化(niche differentiation) 等一系列定量描述种间生态位异同的概念. Hutchinson坚信量化了各物种的生态位大小及种间生态位的 差异, 就可以理解和预测物种分布、多物种共存以及 群落生物多样性形成、维持和变化的规律 ${ }^{[24]}$, 这种集 变量关系描述与逻辑推断于一体的理论框架被后世称 为生态位理论 (niche theory) ${ }^{[25]}$. 简而言之, 生态位理论 基于量化的物种生态位差异, 主要根据竞争排斥原理, 解释和预测物种共存、群落构建、群落结构和功能动 态 $^{[25]}$. 根据同时同地同营养级, 多物种共存的先决条 件是它们所食资源有所差异或被不同消费者所食, Hutchinson预测群落中稳定共存物种的数量小于等于 限制性资源或消费者类型; 之后又用多维资源中生态
位略有差异物种就可稳定共存的逻辑, 解释了少数资 源限制下很多物种共存的现象 ${ }^{[24]}$. 至此, 生态位概念 与特定群落内共存物种的数量多少, 这一生态学的基 本问题紧密关联 ${ }^{[24]}$. 由此可见, 生态位理论从本质上 有别于用统计和数学描述生物与环境关系的早期生态 学研究, 它通过严密的逻辑推断和数学模型, 从物种属 性和环境状况自下而上地解释和预测群落物种组成和 结构动态等生态学模式, 使群落生态学从现象描述转 向假设检验式的科学. 从此, 生态学家们普遍坚信, 基 于物种生态位的确定性作用决定群落物种共存和多样 性动态, 理论生态学家们进而专注于探讨种间生态位 的极限相似性, 实验生态学家则专注于在自然生态系 统中测量和区分各物种的现实生态位 ${ }^{[26]}$. Hutchinson 本人被称为现代生态学之父, 不仅在于他提出了生态 位理论框架和一些生态学根本问题, 奠定了群落生态 学研究的基石, 而且在于他引领和培养了许多杰出的 生态学家继承和发扬了群落生态位理论 ${ }^{[26]}$.

\section{2 群落生态位理论的发展: 以MacArthur的贡献 为代表}

在发展群落生态位理论的路上, Robert MacArthur 引领的工作可能最具代表性和开拓性. MacArthur的贡 献主要基于解决两个群落生态学根本性难题: (i) 如 何基于物种的属性量化生态位; (ii) 物种间生态位的 差异到底要多大, 才能实现多物种稳定共存. 首先, MacArthur发展了种群生活史对策理论, 用物种生活史 对策及其相关的资源利用来量化物种生态位. 1962年, MacArthur将种群密度调节生活史特征的r-K对策思想 引申至物种间生活史对策差异, 提出 $\mathrm{r}-\mathrm{K}$ 对策的演化不 仅受种群密度调节, 而且受长期演化中环境资源限制 性调控, 形成物种水平的 $\mathrm{r}-\mathrm{K}$ 连续体及相关的资源获取 和利用对策 ${ }^{[27]}$, 影响物种在群落中的周转 ${ }^{[28]}$ 以及地理 分布 ${ }^{[29]}$. 其次, MacArthur等人将李雅普诺夫稳定性中 的最小化原则引入Lotka-Volterra模型中, 提出物种共 存的资源利用极限相似性(limiting similarity), 从而量 化物种稳定共存的生态位重叠极限 ${ }^{[30]}$; 预测群落物种 数决定于环境资源能被多少个生态位不过于相似的物 种所分割 ${ }^{[31]}$. 他甚至提及群落动态与物种对策快速演 化的互馈影响: 当群落中已存物种间生态位差异较大 时，新侵入物种趋异演化、生态位介于已存种间; 反 之, 已存种间生态位相似时, 新侵入种在群落中成功 
拓植以生态位趋同于竞争优势者为前提; 群落种间的 紧密程度(species packing)随生产力增大而增加，随生 态位宽度减小而增加，随生态位维度增多而增大 ${ }^{[30]}$. 简而言之, MacArthur及同事将博物学中的生活史对策 理论与生态位概念框架有机结合，基于Lotka-Volterra 竞争模型定量解释和预测了物种生态位及群落生物多 样性动态平衡规律. 通过融合演化生物学与理论生态 学的研究, MacArthur开创了用确定性的生态位作用定 量解释和预测物种分布和群落过程的新纪元, 使群落 生态学理论研究成为生态学的主流.

值得注意的, 是MacArthur也认为生态位确定性作 用未必是物种分布和群落动态的决定性因素. 例如, 他 与 $\mathrm{May}^{[32]}$ 合作提出生态位及其重叠程度并非固定不 变, 而是环境随机波动的函数, 生态位分化可能并非物 种共存和生物多样性变化的必要条件. 这一思想在岛 屿生物地理学理论中体现地更加彻底, 岛屿上物种多 样性并不依赖于物种生态位, 而取决于邻近陆地种库 以及扩散限制 ${ }^{[33]}$; 该理论之后成为种库假说 ${ }^{[34]}$ 、群落 中性理论等生态学核心理论的基础. 从这个意义上讲, MacArthur对随机性作用的强调, 比他所发扬的生态位 理论更具开拓性. 基于同时强调群落构建的生态位确 定性作用和随机过程的重要性, MacArthur等人确立了 群落生态学研究的根本问题——群落构建中的必然性 与偶然性 ${ }^{[1]}$. 可以乐观地想象, 若MacArthur能持续深 究这一基本问题, 人们也许能更清晰地了解什么地方 生态位构建更重要，什么时候扩散限制或随机作用主 导群落动态. 可惜随着MacArthur的英年早逝, 整合演 化生物学、群落生态学与数学的研究并未成为生态学 的主流. 在挖掘Hutchinson和MacArthur等人思想遗产 的基础上，现代生态学家们执迷于强调自己所关注生 态学过程的决定性作用 ${ }^{[35]}$. 资源比例假说将生态位的 确定性作用推至巅峰, 当代共存理论强调多物种共存 不仅以生态位分化为条件而且依赖于种间适合度差 异, 群落中性理论则彻底否定了生态位的重要性.

\section{3 群落生态位理论的兴盛: 资源比例假说和当 代物种共存理论}

20 世纪八九十年代, 正当实验生态学家困惑于, 甚 至怀疑理论生态学所提出的生态位模式解释和过程推 测时，Tilman以精巧的控制实验澄清了单物种和多物 种混播情况下对资源的利用效率，几乎完美地解释了
实验草地群落中植物虽受少数几个营养元素限制, 但 可形成多物种共存的现象. 首先, 基于物种 $\mathrm{r}-\mathrm{K}$ 生活史 对策思想, Tilman ${ }^{[36]}$ 假定物种在㩲取和利用资源上存 在“权衡”差异(物种对一资源消耗能力较强时，对另一 种资源需求则较小), 这种资源权衡导致物种在竞争多 资源时竞争能力存在权衡，产生种间资源依赖的生长 速率和生态位差异. 其次, 根据水域中资源均匀分 布、植物竞争各类资源互不影响等现象(如对氮资源 的竞争不一定影响对磷资源的获取), 他认为, (i ) 陆 地生态系统中景观水平资源分布也较均匀, 而局域群 落中微环境资源异质性较高; (ii) 物种竞争各类资源 互不影响(如地上光竞争与地下土壤资源竞争相对独 立); (iii) 干扰、资源波动等环境变化对各物种资源利 用的影响并不重要 ${ }^{[36]}$. 他进一步预测多物种在竞争利 用多资源时，种特异性的资源消耗程度(consumption rates)差异导致物种间零增长等倾线(zero-growth isoclines)出现交叉重叠, 在只有一种资源限制时, 对该资 源消耗能力最强的物种竞争排除其他物种; 但在多个 资源受限制时，各物种对不同的资源有竞争优势，多 物种实现稳定共存 ${ }^{[37]}$. 他的资源比例假说及实验验证 的重要意义在于: ( i ) 为Hutchinson多维资源生态位 提供了直接证据, 并阐明了资源利用比例差异介导种 间生态位分化, 调控群落动态 ${ }^{[37]}$; (ii) 用物种资源擢 取和竞争能力权衡, 不仅成功解释了群落物种多样性 稳定维持机理(群落内资源比例空间异质性所致), 而 且解释了物种更替和群落演替现象(群落中资源比例 随时间变化所致 $)^{[38]}$; (iii) 将生物对环境资源的需求与 其对环境(生态系统功能)的影响统一, 成功解释了实 验条件下物种多样性对生产力等生态系统功能的影 响, 为澄清生物多样性的作用奠定了基础 ${ }^{[39]}$. 因此, 之 后的30多年中资源比例假说成为生态位理论的代名 词, Tilman引领的工作成为群落生态学研究的旗帜.

然而，生态学界对资源比例假说的质疑从未停止 过. 质疑的焦点不仅在于其前提假设的合理性，也在 其机理在现实自然中的普适性. 例如, 种间竞争能力 权衡的理论依据是个体资源分配权衡原理, 但没有证 据表明这种个体水平的权衡关系可直接推演至种间权 衡 ${ }^{[40]}$; 很多研究也表明, 植物对光、土壤营养等不同 资源的竞争相互影响 ${ }^{[41]}$; 最为典型的Grime-Tilman之 争主要针对资源比例假说在现实群落中的可能性及意

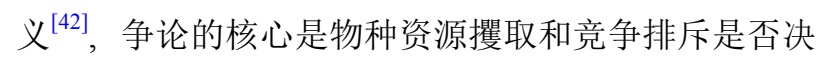


定现实群落动态，其指向目标已不再是资源比例假说 本身, 而是生态位理论的根本一一竞争在物种共存和 群落动态中的主导地位. 基于 CSR三角形对策划分, Grime $^{[43]}$ 认为, 由于干扰、环境波动和资源异质性在 现实自然生态系统中无处不在，与 $r-K$ 对策轴(对应于 R-C对策轴)不相关的耐胁迫(stress tolerators)对策极为 常见, 是群落动态的主要内在调控因素. 然而, CSR三 角形对策本身也是物种适应环境所表现的功能趋同结 果, 用其解释群落结构动态与生态系统功能, 依然是用 低生态水平的结果解释高层次的模式. 与此相似, 2002 年, Westoby等人基于用功能性状量化物种 $\mathrm{r}-\mathrm{K}$ 选择生 活史对策差异, 提出 L-H-S植物生活史主轴 ${ }^{[44]}$ 以及叶 片经济学谱 ${ }^{[45]}$, 但这些描述性划分也类似于Tilman的 种间资源权衡决定群落动态的主张.

在反思竞争和物种生态位分化是否决定物种共存 和群落动态上, 当代共存理论 (modern coexistence theory)尤为典型. Chesson ${ }^{[46]}$ 基于放宽MacArthur消费者资源模型中的假说，提出只有在适合度相同时，种间 生态位分化才是决定共存的必要条件. 简而言之, 物 种稳定共存不仅决定于种间是否存在生态位分化, 而 且取决于其适合度的差异一一生态位的差异作为一种 稳定化机制, 减弱竞争排斥的影响; 适合度差异影响竞 争的不对称性, 可能促进竞争排斥 ${ }^{[46]}$. 关于当代物种 共存理论, 近期本团队 ${ }^{[47]}$ 也做过较详细的介绍, 这里 不再赘述. 需要提及的是, Chesson的贡献在于原创性 强调了适合度差异对物种共存的重要性, 率先指明了 生态位分化的重要性以适合度比较为条件; 进一步提 出了一个探讨群落物种共存的基本框架——稳定化机 制和均等化机制的共同作用和相对重要性，该框架成 为当前物种共存研究的核心主题. 然而, 应质疑其具 体解析方法是否过于晦涩和有效, 甚至是否必要和正 确. 更重要的是, 生态位分化和适合度差异是物种共 存的必要条件, 但是否如Chesson所言是“充要”条件值 得深究 ${ }^{[48]}$. 再者, 如果一切影响群落生物多样性形成 和维持的机制都可归为稳定化机制和均等化机制, Chesson的模型又像是一个笼统的概念框架, 而非具体 解析方法 ${ }^{[48]}$.

\section{4 群落生态位理论的困境: 来自零模型和中性 理论的挑战}

虽然以 Tilman和Chesson为首的主流生态学界强
调物种生态位的决定性作用, 生态学界也始终关注着 MacArthur所强调的扩散限制、环境波动等随机过程 的重要性. 从20世纪七八十年代至今, 生态学家们前赴 后继地用零模型解释群落物种或性状结构时空变

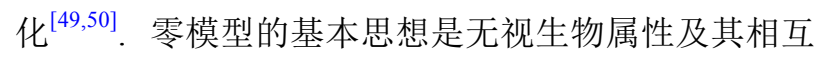
作用的重要性, 用统计学的随机模型解释自然群落中 生物多样性变化; 若能解释, 就说明基于生物属性的 生态位确定性作用在群落动态中不重要, 群落构建由 随机作用主导. 显然, 零模型的方法无论从前提假 设、逻辑递进上还是从机理解释上都经不起推敲. 首 先, 即使零模型可以解释自然群落的物种或性状结构 变化, 也不意味着群落构建过程中物种属性不重要, 生物相互作用就可以被忽略 ${ }^{[51]}$; 例如, 如果环境篮选 导致性状趋同作用可抵消种间竞争导致的性状趋异, 性状分布就会符合零模型预测 ${ }^{[52]}$; 在自然群落动态中 如此相反的生态位作用相互抵消, 导致群落模式符合 零模型预测的现象非常普遍 ${ }^{[53]}$. 其次, 无论如何构建, 零模型总是难以区分各种混合交互作用的生态过 程 ${ }^{[54]}$; 最重要的是, 零模型本身没有机理, 不能回答为 什么零模型能解释自然群落模式这一根本性问题 ${ }^{[51]}$. 这就不难理解, 为什么针对零模型的争议从其提出起 就从未停止过; 我们甚至认为虽然其思想可借鉴, 但 零模型将“不知道”视为“没作用”的逻辑应被抛弃, 至 少不应解读为随机作用的大小. 那么如何定义和量化 群落中的随机作用呢? 群落中性理论提供了一个典范.

基于扩散限制和个体随机漂变的生物学机理, 群 落中性理论继承和发扬了岛屿生物地理学理论, 不仅 彻底否定了物种生态位分化对群落构建的决定性作 用, 而且否定了群落结构动态的平衡性 ${ }^{[5]}$. 关于群落 中性理论假设、逻辑、预测和检验等, 本团队 ${ }^{[1]}$ 此前 有较详细的介绍, 这里不再赘述. 结合本文主旨, 需要 指出的根本问题是Hubbell及其支持者从未澄清过: (i) 为什么个体(因此物种)必然演化出一致的生态功 能; (ii) 为什么自然中存在群落结构动态平衡性和演 替现象. Hubbell曾经以物种资源利用权衡、功能群的 划分忽视功能群内的物种差异等, 来辩解其个体生态 功能一致假设的合理性及可能性 ${ }^{[56]}$, 但至今没有人真 正直接回答个体生态一致性的演化问题. 基于可能的 适合度一致, 近中性理论为放宽中性理论的假设提供 了一种可能 ${ }^{[57]}$. 其次, 中性理论支持者可以举出很多 群落动态非平衡的案例, 声称群落平衡只是一个相对 
概念; 可是, 从早期的群落演替顶级之说到如今的最大 熵群落动态理论 ${ }^{[58]}$, 生态学界从未真正否定过群落的 动态平衡, 也未停止过从动态平衡角度解释和预测各 种物种或性状的组成和相对多度 ${ }^{[59]}$. 与此相应，无法 根据中性理论解释和预测物种/性状的结构, 也就难以 探讨物种对环境的影响 ${ }^{[57]}$. 当然, 统计物理学中经常 提及高层次的群落属性动态与低层次的组分动态之间 也可能无必然的联系. 无论如何, 群落中性理论的成功 (以极少的参数预测了较多的模式), 使人们不得不反 思生态位的决定性作用，重视扩散限制和随机漂变的 重要性. 至少, 在环境适宜的热带雨林等生态系统中, 各物种适合度相似，细微的物种生态位分化就为共存 提供可能，扩散限制下的个体随机漂变可能主导群落 构建 ${ }^{[60]}$. 从这个意义上，无论是适合度相似还是生态 位分化, 都是物种共存和群落多样性维持的必要条件 之一, 而非充要条件. 群落构建不仅决定于生物和非 生物环境选择导致的生态位和适合度差异等生态位确 定性作用，而且取决于环境的随机波动、扩散限制及 个体漂变等随机过程 ${ }^{[61]}$. 当前需要回答的关键科学问 题是基于生态位的确定性作用与随机因素如何“交互 影响”物种共存和群落构建, 更重要的是这些调控群落 动态的基本生态过程可能随尺度、时间和地点变化而 变化, 对此人们也是一无所知 ${ }^{[62]}$.

最后, 生态位理论从提出、兴盛到如今的困境, 始 终将生物对环境的需求和影响作为一枚硬币密不可分 的两面，如 Tilman所提的 $\mathrm{R}$ *既是物种资源需求也是其 影响. 2003年, Chase和Leibold ${ }^{[2]}$ 基于对生态位概念发 展的总结, 强调生物对环境的需求生态位(requirement niche)与其对生态系统功能的影响生态位(impact niche)之间可能并无必然的关联, 二者都是生态位的不 同组分, 他们将此称为当代生态位理论. 越来越多的研 究表明, 对物种共存和群落结构动态有重要影响的需 求生态位差异, 可能对生态系统功能的影响并不重要, 反之亦然. 例如, 种间比叶面积指数(specific leaf area, SLA)的差异可指示物种资源㩲取能力的差异，但物种 或群落的生物量积累与SLA相关性较弱, 而由叶片干 物质含量(leaf dry-matter content, LDMC) 所决定 ${ }^{[63]}$, 在局域群落中物种LDMC和SLA之间也并无强的负相 关关系 ${ }^{[64]}$. 基于此, 2002年, Lavorel和Garnier ${ }^{[63]}$ 提出了 响应-影响性状框架(response-effect trait framework)将 基于物种对环境的需求与其影响分离, 并推演至群落
功能属性动态和生态系统功能变化. 该框架很好地描 述了一些性状影响群落功能属性变化而另一些性状决 定生态系统功能; 但实际中很多性状(如植物高度)既 是响应性状也是影响性状，区分二者并不现实; 该框 架也未与物种共存和群落构建理论有机结合, 成为可 预测性的理论体系.

总之, 历经半个多世纪的发展, 生态位概念及思想 在预测物种分布等大尺度生物地理学过程中被广泛应 用，发展成流行的生态位模型 ${ }^{[65]}$; 但在其发源的群落 生态学中, 并未揭示出学界认可的物种共存及群落构 建的一般性机理. 反而越来越明多的研究表明, 生态 位并非物种固有属性，生态位分化也非物种共存的充 要条件, 生态位确定性作用不一定是群落构建的驱动 力. 如此诸多生态位“不是什么”的讨论, 使人们不禁 追忆起Grinnell和Elton的生态位初义, 再思Hutchinson 生态位及生态位理论的合理性和有效性.

\section{3 生态位理论困境的根源：再思生态位是 什么}

随着Hutchinson用多维环境资源量化物种生态 位、构建生态学理论, 生态学家们前赴后继, 不解努力 地量化各种环境中物种生态位, 以期揭示物种适合度 动态及群落构建机理. 但至今有共识的机理性解释和 预测却少之又少, 生态位理论似乎更像是一个后验式 的解释框架, 被广泛用以解说各种自然现象, 但又难 为解析方法, 合理而有效地预测任何机理和模式. 这 不得不使人们开始怀疑在探讨生态位的路上，是否走 叉了方向, 生态位是什么、是否可以量化, 生态位分 化到底是原因还是结果, 甚至怀疑生态位存在的真 实性。

\section{1 生态位是群落动态的驱动原因, 还是涌现结果}

从Hutchinson将生态位视为物种的属性以来, 主 流生态学界似乎始终坚信Hutchinson的还原论逻辑 ${ }^{[66]}$ : 群落动态可以解构为组分种的动态 (及其相互作用), 后者取决于长期演化形成的物种生活史对策，而生活 史对策又可用与物种属性及存活相关的环境变量所刻 画. 因此, 量化物种属性及其与环境的关系, 就可以量 化物种生态位，并基于此自下而上地解释和预测物种 共存和群落动态, 揭示群落生态学的基本规律. 在此 
思想指导下，人们一直遵循从低生态层次的结构和功 能(如物种属性和作用)变化揭示高层次群落动态的逻 辑路线. 但是, 当再深思决定个体或物种属性及功能的 内外因素时，人们不禁怀疑历史环境和自然选择塑造 的决定性作用: 演化生物学和行为生态学的大量研究 表明，物种适合度和性状演化不仅取决于系统发育限 制，而且深受个体对当前环境的响应和适应 ${ }^{[22,67]}$. 从 这个角度，是否可推断物种的性状发育和形成以及生 态功能深受所处(群落)环境的调控呢? 迅速发展的生 态-发育生物学(ecological developmental biology, ecodevo)已经充分表明，个体性状结构的发育和形成是演 化约束下基因表达与个体内外环境互馈影响的结 果 ${ }^{[68]}$; 越来越多的生态-演化动态(eco-evolutionary dynamics, eco-evo)研究也表明, 当前(群落)环境差异驱动 种群遗传多样性、快速演化和个体差异性 ${ }^{[69]}$. 这些个 体发育、性状可塑性及种群适应性导致的种内性状变 异，并不一定远小于种间性状差异 ${ }^{[70]}$, 在大部分动植 物中种内差异与种间差异相当 ${ }^{[71]}$, 在环境较严酷的生 境中甚至大于种间变异 ${ }^{[72]}$, 介导群落功能结构动态 ${ }^{[73]}$. 因此，忽视环境反馈调控生物属性和种内个体生态差 异的Hutchinson生态位及其理论，无论从逻辑上(物种 生态位: 种间 >>种内差异)还是在实践中都遇到严峻 的挑战 ${ }^{[70]}$ ，一些学者因此认为Hutchinson生态位应该 被学界所抛弃 ${ }^{[5]}$. 基于个体的生态学模型迅速发展, 为 整合个体间生态差异的作用提供了新思路，不少生态 学家甚至致力于发展个体生态位及理论 ${ }^{[7]}$. 当然, 如 果每个个体都具有随群落环境和自身行为等变化的生 态位, 生态位概念也就没有任何实质意义; 并且完全否 认个体、种群等低生态层次的共性也不符合科学逻辑 和实际. 人们所需的是结合生态、演化、发育和行为 研究, 再思(群落)环境对个体属性及适合度影响, 如何 反映至物种生态位变化, 后者又如何反馈影响至群落 动态.

在探讨整体群落环境对其组分影响方面，整体论 的视角在中国传统文化中有着悠久的历史，在西方生 态学发展早期也有群落有机体与个体论之争, 之后也 有Odom的系统生态学. 只是自Hutchinson时代至今, 整体论思想在群落生态学研究中并未受到足够重 视 ${ }^{[75]}$. 但是Brown等人 ${ }^{[76]}$ 引领的宏观生态学研究从能 量分布的维度成功解释了群落生物多样性宏观模式, 提出生产力等生态系统功能可能决定局域群落生物多
样性. 这种自上而下式群落功能决定结构的探索为群 落生态学提供了另一种思路. 这里, 不得不提及生态 位中文翻译者，王刚老师对生态位的重新定义及发展 尝试. 1984年, 王刚等人 ${ }^{[77]}$ 应用数学中的集合映射理 论，提出广义的生态位是多元环境(包括种间关系)向 量集与物种(属性集)的映射关系; 物种生态位宽度可 用使物种多度大于零的环境因子变幅来度量; 生态位 体积便是映射关系的多重积分, 表示物种对多维环境 因子的综合适应能力; 种间生态位重叠可用物种与多 元环境联系中的相似性来量化. 他进一步指出, 多维 环境资源决定(植物)群落分布(梯度)序列，即“群落 位” (coenoniche $)^{[78]}$, 群落位可被群落中出现的组分种 瓜分, 形成各物种的生态位、生态位重叠以及适合度 (图2), 类似于MacArthur的折棒模型 ${ }^{[79]}$. 这一自上而下 的生态位及适合度预测, 为人们从整体群落环境中量 化物种生态位提供了另一种思路, 可惜之后并未引起 学界的重视, 也未被继承和发扬. 与此相似的是, 2005 年, Shipley等人 ${ }^{[80]}$ 以环境变量计算草地演替中群落水 平性状，用后者进一步成功预测了各演替阶段物种相 对多度及其变化. 随之, 他将此统计方法发展为群落 构建的最大熵理论, 提出非生物环境决定群落基本属 性，群落基本属性进而决定优势的性状和物种，稀有 的性状和物种则取决于随机过程 ${ }^{[81]}$. 在更宏观的层面, 2011年，Harte也用最大熵解释和预测了种面积曲线等 生态学模式 ${ }^{[82]}$, 他最近将此发展成动态最大熵模型 ${ }^{[58]}$. 虽然这些侧重自上而下式预测群落动态和物种适合度 的研究也存在诸多问题(如群落基本属性源自何处、 最大熵原理是否适应于开放的自然系统等); 但这些从 整体到组分的理论和方法在预测群落结构和生物多样

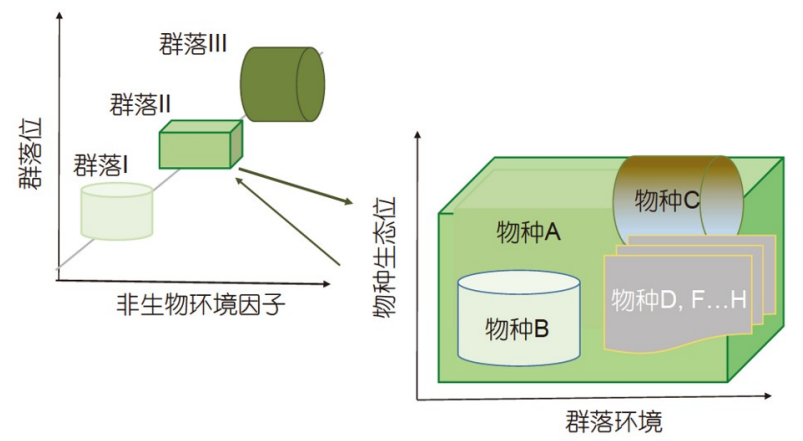

图 2 群落位与物种生态位的关联示意图

Figure 2 Diagram of linkage between coenoniche and species ecological niche 
性动态方面，似乎比从组分到整体群落的生态位理论 更加可行和有效, 为人们认识生态位的本质及重建群 落生态学理论提供了新方向.

\section{2 生态位既是群落动态的驱动因素, 更是物种适 应群落环境的结果}

不可否认生态位是有机体属性相关的物种对环境 的需求和影响, 这些需求和影响是物种共存、群落构 建等群落过程的重要驱动作用. 但另一方面, 如果自 上而下 (从群落到组分种)解释和预测物种适合度的逻 辑合理，不难发现生态位更像是物种适应群落环境的 涌现结果 ${ }^{[75]}$, 而非驱动物种共存和群落动态的根本原 因. 实际上，从个体层面的性状发育、物种生活史对 策权衡、群落自组织、生态系统生产力形成至地球本 身的平衡状态维持, 学界从未停止过思索高级生态层 次(如群落)的属性和功能决定低层次结构的可能性. Huxley ${ }^{[83]}$ 早在 19 世纪就指出, 有机体在其生长发育过 程中实现性状分化(快速演化), Smith和Szathmary ${ }^{[84]}{ }^{\text {等 }}$ 现代演化生物学家们进一步明确个体性状的形成和作 用主要受控于遗传信息随环境状况的表达，而不仅是 遗传信息本身. 基于此思想, 干细胞的生态位(stem cell niche)被定义为确定干细胞命运又被细胞反馈影响的 微环境, 强调了生态位既是生命活动的原因也是结 果 ${ }^{[85]}$. 与此相似，盖亚假说(Gaia hypothesis)也强调宏 观生命与环境互为因果演化, 维持地球的平衡状态 ${ }^{[86]}$; 自组织模型(self-organization model)也常被用以探讨 群落和生态系统演化中结构和功能的互馈作用 ${ }^{[87]}$. 群 落生态学中, 虽然主流观点坚信生物多样性决定生产 力，但是无论是宏观尺度上生物多样性地理格局的能 量假说 ${ }^{[76,88]}$, 还是普遍存在的施肥导致生物多样性丧 失事实，都提示生产力决定生物多样性的可能. 一些 学者甚至认为生物多样性与生产力的关系, 如鸡和蛋 的哲学问题，难以阐明. 借鉴干细胞生态位和盖亚假 说的思想, 不难想象生物多样性与生产力也是互馈演 化的关系, 而非谁决定谁的问题.

据此，可以肯定物种生活史对策和生态位也并非 固定不变, 而是受群落环境变化与个体适应互馈影响 而快速变化 ${ }^{[27,35]}$. 问题是人们可能高估长期演化对有 机体生态对策的决定作用，而低估了生物响应环境， 以性状可塑性、行为等调整其生态对策，影响适合度 和生态位, 进而影响群落过程. 例如, 物种生态位分化
的根据是生活史对策权衡，但几乎所有的权衡都可以 被生物性状可塑性、快速演化和行为所弱化或克服; 物种水平的性状权衡更多是物种特异地适应所处环境 的结果, 而非决定物种生态对策的原因 ${ }^{[40]}$. 总之, 当考 虑到当前群落环境对生物属性形成的深刻影响时, 不 难发现生态位也是物种适应所经历环境的结果. 从这 个意义上, 不难理解为什么生态位能解释几乎所有的 生态现象, 又难以预测任何机理和模式一一用既成的 低生态层次结果(物种性状和生态位模式)循环论证式 解释更高层次的群落学结果. 至少可以肯定的是, 生态 位并非如Hutchinson所称的物种固有属性, 也非群落 动态的唯一根本驱动力; 它既是群落环境影响生物属 性和适合度的结果, 又是生物反馈影响群落过程的驱 动因子.

\section{4 动态生态位: 一个理解群落生态学规律 的新框架}

既然生态位也像是群落构建的结果, 那么是否还 有必要沿用生态位这个基本概念呢? 我们认为，当前 没有更好替代概念刻画生态学意义下有机体(物种)属 性，生态位概念依然必不可少。不该因生态位概念的 抽象性及其理论的不确定性, 而完全抛弃生态位; 而 应回归生态位概念的初义, 从生物与环境互馈演化角 度, 整合随机过程(环境的随机波动及个体的随机漂 移)对物种适合度动态和群落构建的影响, 重新审 视、发展和更新生态位概念(图1), 以修订或重建群 落生态学理论.

\section{1 生态位概念难为解析方法, 又是必要的概念 框架}

如上所述, Hutchinson对生态位的量化借鉴了数 学中的(二元)对偶原则(duality principle), 将影响生物 的多资源环境与生物的多维属性看作群落环境-生态 位对偶体(biotope-niche duality). 但不知为何Hutchinson及其支持者并未特别说明数学中对偶关系并非总 是一一对应, 生物学中一一对应的环境-生态位也并不 常见 ${ }^{[16]}$. 在随时随地变化的环境中, 量化Hutchinson生 态位既不符合数学逻辑, 也不具现实意义. 例如, 在一 块裸露的草地斑块中, 可能本无植物生态位, 当 $\mathrm{A} 、 \mathrm{~B}$ 和 $\mathrm{C}$ 三种种子扩散至此, 萌发、生长和繁殖时, 能存活 
的植物就影响并改变了当地微环境, 逐步形成植物、 微生物, 甚至食草动物生态位, 这个过程就是所谓的 生态位构建(niche construction) ${ }^{[89]}$. 在这个环境和生物 互馈影响变化的过程中, 量化某一时间段某种生物的 (瞬时)生态位, 也无异于刻舟求剑, 不能预测其未来适 合度. 若量化各种植物对资源的需求及对其他生物的 影响，预测 $\mathrm{A}$ 种植物逐步成为优势种而 $\mathrm{C}$ 种植物消失 时，干旱等偶然的环境波动又有利于 $\mathrm{C}$ 种植物，使 $\mathrm{A}$ 或 $\mathrm{B}$ 种植物减少或消失, 为新入侵者 $\mathrm{D} 、 \mathrm{E} 、 \mathrm{~F}$ 植物提供了 生态位, Tilman ${ }^{[90]}$ 将此称为随机生态位. 从这个意义 上，人们所看到的某时间截面群落多物种共存现象, 更多地是少数种的生态位随环境演化, 混杂着多数种 随机呈现的瞬间; 尽管模式上类似生态位格局，群落 构建可能不是由生态位确定性作用所主导; 反之亦然, 各种基于生态位的确定性作用交互影响也可能会形成 中性格局 ${ }^{[91]}$. 因此，若不能刻画出生态位随时空环境 变化的规律, 量化瞬时生态位并基于此预测群落动态 的现实意义将非常有限, Hutchinson生态位也就难成 为探讨群落生态学规律的解析方法.

不能作为解析方法是否意味着可以完全抛弃生态 位概念及其框架呢？随着应用生态学的兴起及群落生 态学理论的衰退，过去30年生态位一词在文献中的出 现频率持续下降 ${ }^{[3]}$ ，一些学者甚至呼吁抛弃生态位概 念及理论, 如最近对Hutchinson现实生态位的否定 ${ }^{[5]}$. 当然，如果有新的概念能逐步替代生态位对物种生态 学作用和影响的刻画, 有新理论可覆盖生态位理论所 探寻的主题，这种抛弃也无可厚非。可惜根据相关文 献，并没有新的相关概念和理论能够替代生态位及其 理论, 得到学界重视和讨论 ${ }^{[3,4]}$. 理论生态位学家们依 然执迷于用描述性的Lotka-Volterra种间竞争模型和经 典生态位框架, 探讨群落动态的一般性规律; 大数据科 学背景下的实验生态学家们则热忱于以大数据报道各 种观测变量之间的相关性 ${ }^{[3]}$ ，这种状态似曾相识似乎又回到了 20 世纪上半叶的生态学. 这次所不同的 是用遗传或性状多样性替代了物种多样性，网络相关 方法和思想深入人心. 虽然越来越多的研究使人们对 生态位确定性作用的认识更加具体细节，很多新的方 法也揭示了生态位构建往往并不起决定性作用 ${ }^{[35]}$; 但 很少有研究真正颠覆传统生态位理论的基本框架，为 追寻一般性规律指明新的方向. 例如，用功能性状的 研究更多是量化物种属性和生活史对策, 很少有研究
基于种内变异揭示生态-演化作用如何影响物种生态 位动态与群落过程 ${ }^{[92]}$. 总之, 在没有新的替代性概念 和理论之前, 抛弃生态位概念及理论框架, 势必使人 们失去方向; 但是, 再多各种特异性现象的报道和相 关性的详实描述，也难总结出一般性规律一一真如物 理学家常常讱讽生物学家描述了各种水果落地, 也不 可能总结出万有引力. 因此, 我们认为生态位概念框 架依然必不可少，当前急需在反思问题的基础上，整 合新的研究进展和认识, 更新生态位概念, 发展新的 生态位相关理论.

\section{2 整合随机作用和生物-环境互馈演化的动态生 态位}

回溯生态位理论发展的历程, 我们获得的最大感 悟可能莫过于真实的生态学过程是确定性生态位作 用与随机过程(包括环境随机波动和生物随机漂变)交 互影响的结果; 从有机体的存活, 到物种共存和群落 构建等各生态层次的动态无不受随机作用深刻影 ${ }^{\text {响 }}{ }^{[1,91]}$. 在演化生物学中, 对这种环境依赖的生物过程 及其不确定性的认识和量化已近百年. 1932年, Wright ${ }^{[93]}$ 就把基因随环境表达对适应度影响的不确 定性描述为适应度景观(fitness landscape), 之后, 分析 动态适合度(dynamic fitness)成为演化生物学的主题. 借鉴于此, 是否可以用动态的(dynamic)视角重新审视 生态位概念呢? 干细胞的动态生态位(dynamic stem cell niche)被笼统地描述为干细胞周围动态变化的非 生物环境, 对干细胞特性的维持或分化起到决定性作 用, 也受干细胞活动的反馈影响 ${ }^{[94]}$. 这一定义与生态 学中生态位概念的本质区别在于强调了环境与生物 互馈影响、动态变化. 一些生态学文献也提及动态的 生态位分割(dynamic niche partitioning) ${ }^{[95]}$ 、生态位 的时空动态(niche dynamics) ${ }^{[96]}$ 、生态位演化(niche evolution)等生态位的变化属性，但这些提法依然强 调生态位为生物(或环境)固有属性，而后受演化影响， 缓慢变化 ${ }^{[96]}$, 本质上有别于本文要突出动态生态位在 生物与环境互馈影响中有无相生、在动态变化中 形成.

根据生态位初义, 此处定义动态生态位(dynamic niche)为生物与环境互馈影响的动态潜在关系(需求和 影响); 它不仅取决于有机体的属性及其变化、基于属 性的生物与生物相互作用、生物与环境的互馈影响, 
而且深受随机过程调控. 数学上, 动态生态位可表示为 有机体的生物属性 $(\mathrm{T})$ 及环境因素( $\mathrm{E})$ 影响其多度变化 $(d \mathrm{~F})$ 的 (联合) 概率函数(可表示为: $d \operatorname{Niche}(T, E)$ ); 多度 变化可表示为动态生态位的概率 $P(d \mathrm{~F})$, 不仅受生物与 生物及环境的(生态位)确定性相互作用影响, 而且受 环境随机波动、扩散及个体漂变的深刻影响(以概率 表达). 用一个三维图示意(图3), 此处将动态生态位概 念框架释义为: ( i ) 基于Grinnell生态位初义, 用有机 体的性状与其周围环境的关系, 表示动态变化的生物 属性(性状结构)对环境的需求及影响; (ii) 基于Elton 生态位强调生物与生物相互作用, 用有机体性状与多 度变化的关系表示生物属性对其自身适合度及其他生 物的影响, 以及被反馈影响, 即环境依赖的性状与适合 度关系问题; (iii) 与Hutchinson等前人强调的生态位有 本质区别, 此处强调有机体的多度(或适合度)变化不 仅取决于环境介导的生态位确定性作用, 而且深受环 境随机波动及个体扩散、迁入/迁出等随机过程影响, 导致确定性作用对多度(或适合度)的决定性以概率形 式呈现; 当然, 随机作用的重要性也与群落系统属性和 大小(如群落性状结构和个体多度)密切相关. 简而言 之, 此处用有机体(物种)性状分布及其变化, 表征生物 属性和适合度变化与环境互馈影响; 用确定性作用的 概率表达, 强调了随机过程对物种适合度、生物相互 作用和群落构建的影响. 更进一步地, 通过将“有机 体” 视为个体、种群、群落, 动态生态位的概念甚至可 以扩展至各生态水平, 以及从个体属性变化至群落过 程关联的探讨.

应用动态生态位概念框架方面，可以借鉴贝叶斯 概率方法 $[P(d \mathrm{~F})=P(\mathrm{E}) P(\mathrm{~T} \mid \mathrm{E})=P(\mathrm{~T}) P(\mathrm{E} \mid \mathrm{T})]$, 探讨各生态 层次的生物与环境互馈关系. 例如, 若将群落视为有机 体, 局域总体非生物环境就可作为影响群落过程的全 概率 $[P(\mathrm{E})]$, 特定局域环境中群落的功能属性 $\left[P\left(d \mathrm{~F}_{\mathrm{c}}\right)\right.$ 、生产力等 $]$ 与群落性状结构 $\left[P\left(\mathrm{~T}_{\mathrm{c}} \mid \mathrm{E}\right)\right]$ 的关系, 可作为群落的动态生态位问题 $\left[P\left(d \mathrm{~F}_{\mathrm{c}}\right)=P(\mathrm{E}) P\left(\mathrm{~T}_{\mathrm{c}} \mid \mathrm{E}\right)\right]$; 若将物种属性 $\left[P\left(\mathrm{~T}_{\mathrm{sp}}\right)\right]$ 作为全概率, 探讨特定群落环境 中该物种种群性状 $\left[P\left(\mathrm{~T}_{\mathrm{sp}} \mid \mathrm{E}_{\mathrm{c}}\right)\right]$ 与其个体多度 $\left[P\left(d \mathrm{~F}_{\mathrm{sp}}\right)\right]$ 的 概率关系, 就可了解物种的动态生态位 $\left[P\left(d \mathrm{~F}_{\mathrm{sp}}\right)=P\left(\mathrm{~T}_{\mathrm{sp}}\right)\right.$ $\left.P\left(\mathrm{~T}_{\mathrm{sp}} \mid \mathrm{E}_{\mathrm{c}}\right)\right]$; 同样, 探讨某基因型或表型的适合度与环境 的关系时, 可将该基因型 $\left[P\left(\mathrm{~T}_{\text {gene }}\right)\right]$ 或表型 $\left[P\left(\mathrm{~T}_{\text {phen }}\right)\right]$ 作 为全概率, 量化特定群落环境中该基因型 $\left[P\left(\mathrm{~T}_{\text {gene }} \mid \mathrm{E}_{\mathrm{c}}\right)\right]$

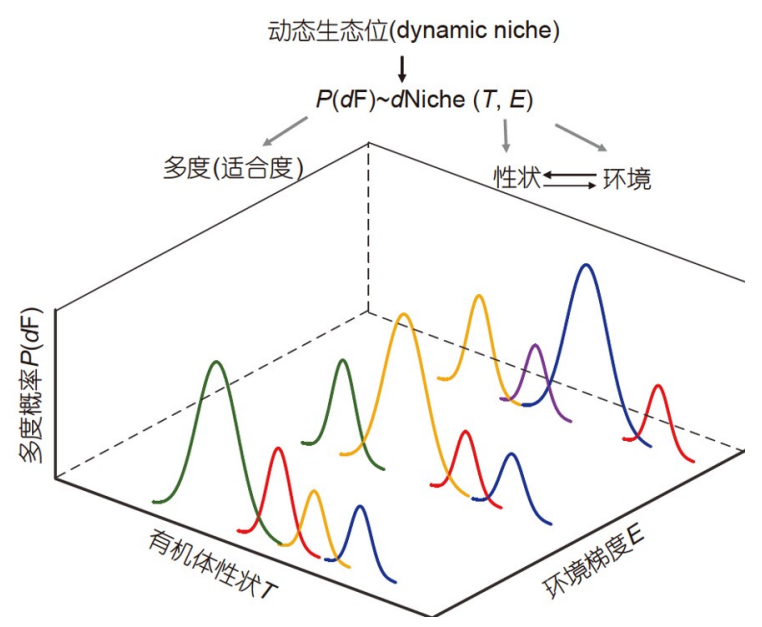

图 3 动态生态位概念框架示意图

Figure 3 Diagram of the conceptual framework of dynamic niche

或表型 $\left[P\left(\mathrm{~T}_{\text {phen }} \mid \mathrm{E}_{\mathrm{c}}\right)\right]$ 与其适合度 $\left(P\left(d \mathrm{~F}_{\text {gene }}\right)\right.$ 或 $\left.P\left(d \mathrm{~F}_{\text {phen }}\right)\right)$ 的 关系, 以探讨该基因型或表型的动态生态位. 结合自上 而下整体论思想与自下而上的还原论推演, 也可以探 讨有机体性状变化和存活状况对群落过程的影响以及 群落过程对有机体的影响. 例如, 若将特定环境下群落 (性状)属性作为全概率 $\left[P\left(\mathrm{~T}_{\mathrm{c}} \mid \mathrm{E}_{\mathrm{c}}\right)\right]$, 探讨该群落中各物 种的多度动态 $\left[P\left(d \mathrm{~F}_{\mathrm{sp}} \mid \mathrm{T}_{\mathrm{c}}\right)\right]$ 与群落环境 $\left[P\left(\mathrm{E}_{\mathrm{c}} \mid \mathrm{T}_{\mathrm{c}}\right)\right]$ 的关系, 就可讨论群落环境对物种多度的影响, 以及物种对群 落属性和功能的反馈作用.

总之, 通过将物种的属性及适合度拓展表达为变 化环境下的性状分布, 动态生态位概念框架整合了生 物与环境间的互馈作用对物种生态位变化的影响. 通 过探讨生物属性和环境交互作用对有机体适合度的 概率性影响, 以及生物存活及适合度变化对有机体性 状分布和群落环境的影响, 人们进而可讨论随机过程 影响下, 确定性的生物与环境及其他生物互馈影响大 小, 以及随机作用大小对确定性生态位作用的依赖. 在此基础上, 再论动态的生态位决定性作用、环境 波动、扩散和个体漂变等随机作用如何交互调控物 种共存和群落构建, 或自上而下探讨群落功能属性变 化如何影响随机作用和各组分种的生态位动态. 如 此, 随着实践中对具体解析方法的改进和完善, 相信 动态生态位可为继承和发扬生态位思想, 为重构群落 生态学理论奠定基础, 提供新的概念框架和解析 方法. 
1 Niu K C, Liu Y N, Shen Z H, et al. Community assembly: the relative importance of neutral theory and niche theory (in Chinese). Biodivers Sci, 2009, 17: 579-593 [牛克昌, 刘怿宁, 沈泽昊, 等. 群落构建的中性理论和生态位理论. 生物多样性, 2009, 17: 579-593]

2 Chase J M, Leibold M A. Ecological Niches: Linking Classical and Contemporary Approaches. Chicago: University of Chicago Press, 2003

3 McCallen E, Knott J, Nunez-Mir G, et al. Trends in ecology: shifts in ecological research themes over the past four decades. Front Ecol Environ, 2019, 17: 109-116

4 Sales L P, Hayward M W, Loyola R. What do you mean by "niche"? Modern ecological theories are not coherent on rhetoric about the niche concept. Acta Oecol, 2021, 110: 103701

5 Angilletta M J, Sears M W, Levy O, et al. Fundamental flaws with the fundamental niche. Integr Comp Biol, 2019, 59: 1038-1048

6 Johnson R H. Determinate Evolution in the Color-Pattern of the Lady-Beetles. Washington: Comegie Znstitution of Washington Public, 1910

7 Grinnell J. The niche relationships of the California thrasher. Auk, 1917, 21: 364-382

8 Grinnell J. Geography and evolution. Ecology, 1924, 5: 225-229

9 Grinnell J. A distributional Summation of the Ornithology of Lower California. California: University of California Publications in Zoology, 1928

10 Elton C S. Animal Ecology. London: Sidgwick \&Jackson, 1927

11 Li D Z, Liu K Y, Zang R G, et al. Development of the modern niche theory and its main representative genres (in Chinese). Sci Silv Sin, 2006, 42: 88-94 [李德志, 刘科轶, 藏润国, 等. 现代生态位理论的发展及其主要代表流派. 林业科学, 2006, 42: 88-94]

12 Dice L R. Natural Communities. Michigan: University of Michigan Press, 1952

13 Clarke A S. Studies on the life cycle of the pseudophyllidean cestode Schistocephalus solidus. Proc Zool Soc London, 1954, 124: 257-302

14 Whittaker R H, Levin S A, Root R B. Niche, habitat, and ecotope. Am Natist, 1973, 107: 321-338

15 Peterson A T, Soberón J, Pearson R G, et al. Ecological Niches and Geographic Distributions. Princeton: Princeton University Press, 2011

16 Colwell R K, Rangel T F. Hutchinson's duality: the once and future niche. Proc Natl Acad Sci USA, 2009, 106: 19651-19658

17 Taylor W P. The Status of the Beavers of Western North America with a Consideration of the Factors in Their Speciation. California: University of California, 1916

18 Gause G F. The Struggle for Existence. Baltimore: Williams and Wilkins Company, 1934

19 Park T. Interspecies competition in populations of Trilobium confusum duval and Trilobium castaneum herbst. Ecol Monogr, 1948, 18: 265-307

20 L'Héritier P, Teissier G. Recherches sur la concurrence vitale. Etude de populations mixtes de Drosophila melanogaster et de Drosophila funebris. Compt Rendus de la Soc de Biol, 1935, 118: 1396-1398

21 Hutchinson G E. Concluding remarks. Cold Spring Harb Symp Quant Biol, 1957, 22: 415-427

22 Odum E P. Fundamentals of Ecology. 2nd ed. Philadelphia: W.B. Saunders Company and Toppan Company, 1959

23 Hutchinson G E. An Introduction to Population Biology. Connecticut: Yale University Press, 1978

24 Hutchinson G E. Homage to Santa Rosalia or why are there so many kinds of animals? Am Natist, 1959, 93: 145-159

25 Vandermeer J H. Niche theory. Annu Rev Ecol Syst, 1972, 3: 107-132

Skelly D K, Post D M, Smith D S. The Art of Ecology: Writings of G. Evelyn Hutchinson. Connecticut: Yale University Press, 2011

MacArthur R H. Some generalized theorems of natural selection. Proc Natl Acad Sci USA, 1962, 48: 1893-1897

MacArthur R, Levins R. Competition, habitat selection, and character displacement in a patchy environment. Proc Natl Acad Sci USA, 1964, 51: $1207-1210$

29 MacArthur R H. Geographical Ecology: Patterns in the Distribution of Species. Princeton: Princeton University Press, 1972 


$$
\text { 2002, 33: 125-159 }
$$

\section{等. 物种共存理论研究进展. 生物多样性, 2017, 25: 345-354]}

48 Barabás G, D’Andrea R, Stump S M. Chesson's coexistence theory. Ecol Monogr, 2018, 88: 277-303

49 Connor E F, Simberloff D. The assembly of species communities: chance or competition? Ecology, 1979, 60: 1132

50 Kraft N J B, Valencia R, Ackerly D D. Functional traits and niche-based tree community assembly in an Amazonian forest. Science, 2008, 322: $580-582$

51 Gotelli N J, McGill B J. Null versus neutral models: what's the difference? Ecography, 2006, 29: 793-800

52 de Bello F. The quest for trait convergence and divergence in community assembly: are null-models the magic wand? Glob Ecol Biogeogr, 2012, 21: 312-317

53 McGill B J. A test of the unified neutral theory of biodiversity. Nature, 2003, 422: 881-885

\section{1-2363}

63 Ecol, 2002, 16: 545-556

64

$$
\text { Ecography, 2017, 40: 685-697 }
$$

65 Qiao H J, Huang J H, Huang J H. Theoretical basis, future directions, and challenges for ecological niche models (in Chinese). Sci Sin Vitae, 2013, 43: 915-927 [乔慧捷, 胡军华, 黄继红. 生态位模型的理论基础、发展方向与挑战. 中国科学: 生命科学, 2013, 43: 915-927]
MacColl A D C. The ecological causes of evolution. Trends Ecol Evol, 2011, 26: 514-522

Gilbert S F. Ecological developmental biology: developmental biology meets the real world. Dev Biol, 2001, 233: 1-12

Hendry A P. Eco-evolutionary Dynamics. Princeton: Princeton University Press, 2016 
70 Bolnick D I, Amarasekare P, Araújo M S, et al. Why intraspecific trait variation matters in community ecology. Trends Ecol Evol, 2011, 26: 183192

71 Des Roches S, Post D M, Turley N E, et al. The ecological importance of intraspecific variation. Nat Ecol Evol, 2018, 2: 57-64

72 Niu K C, Zhang S T, Lechowicz M J. Harsh environmental regimes increase the functional significance of intraspecific variation in plant communities. Funct Ecol, 2020, 34: 1666-1677

73 Niu K C, He J S, Lechowicz M J. Grazing-induced shifts in community functional composition and soil nutrient availability in Tibetan alpine meadows. J Appl Ecol, 2016, 53: 1554-1564

74 Layman C A, Newsome S D, Gancos Crawford T. Individual-level niche specialization within populations: emerging areas of study. Oecologia, 2015, 178: 1-4

75 Mcinerny G, Etienne R S, Higgins S. Pitch the niche - taking responsibility for the concepts we use in ecology and species distribution modelling. J Biogeogr, 2012, 39: 2112-2118

76 Brown J H, Gillooly J F, Allen A P, et al. Toward a metabolic theory of ecology. Ecology, 2004, 85: 1771-1789

77 Wang G, Zhao S L, Zhang P Y, et al. On the definition of niche and the improved formula for measuring niche overlap (in Chinese). Acta Ecol Sin, 1984, 4: 119-127 [王刚, 赵松岭, 张鹏云, 等. 关于生态位定义的探讨及生态位重叠计测公式改进的研究. 生态学报, 1984, 4: 119-127]

78 Wang G. The coenoniche of plant communities (in Chinese). Pratacult Sci, 1990, 7: 52-56 [王刚. 植物群落的群落位. 草业科学, 1990, 7: 52$56]$

79 MacArthur R H. On the relative abundance of bird species. Proc Natl Acad Sci USA, 1957, 43: 293-295

80 Shipley B, Vile D, Garnier E. From plant traits to plant communities: a statistical mechanistic approach to biodiversity. Science, 2006, 314: 812814

81 Shipley B. From Plant Traits to Vegetation Structure: Chance and Selection in the Assembly of Ecological Communities. Cambridge: Cambridge University Press, 2010

82 Harte J. Maximum Entropy and Ecology: A Theory of Abundance, Distribution, and Energetics. Oxford: Oxford University Press, 2011

83 Huxley T H. Upon animal individuality. In: Proceedings of the Royal Institution. London. 1852

84 Smith J M, Szathmary E. The Major Transitions in Evolution. Oxford: Oxford University Press, 1995

85 Li L, Xie T. Stem cell niche: structure and function. Annu Rev Cell Dev Biol, 2005, 21: 605-631

86 Lovelock J. Gaia: A New Look at Life on Earth. Oxford: Oxford University Press, 2000

87 Ge Z P, Liu Q X. More than the sum of its parts: self-organized patterns and emergent properties of ecosystems (in Chinese). Biodivers Sci, 2020, 28: 1431-1443 [葛振鹏, 刘权兴. 整体大于部分之和: 生态自组织斑图及其涌现属性. 生物多样性, 2020, 28: 1431-1443]

88 Wang Z H, Tang Z Y, Fang J Y. The species-energy hypothesis as a mechanism for species richness patterns (in Chinese). Biodivers Sci, 2009, 17: 613-624 [王志恒, 唐志尧, 方精云. 物种多样性地理格局的能量假说. 生物多样性, 2009, 17: 613-624]

89 Odling-Smee F J, Laland K N, Feldman M W. Niche construction. Am Natist, 1996, 147: 641-648

90 Tilman D. Niche tradeoffs, neutrality, and community structure: a stochastic theory of resource competition, invasion, and community assembly. Proc Natl Acad Sci USA, 2004, 101: 10854-10861

91 Purves D W, Pacala S W. Ecological drift in niche-structured communities: neutral pattern does not imply neutral process. In: Biotic Interactions in the Tropics: Their Role in the Maintenance of Species Diversity. Princeton: Princeton University, 2005

92 Mcpeek M A. Evolutionary Community Ecology. Princeton: Princeton University Press, 2017

93 Wright S. The roles of mutation, inbreeding, crossbreeding, and selection in evolution. Proc Sixth Int Congr Genet, 1932, 1: 359-366

94 Voog J, Jones D L. Stem cells and the niche: a dynamic duo. Cell Stem Cell, 2010, 6: 103-115

95 Guderle M, Bachmann D, Milcu A, et al. Dynamic niche partitioning in root water uptake facilitates efficient water use in more diverse grassland plant communities. Funct Ecol, 2018, 32: 214-227

96 Pearman P B, Guisan A, Broennimann O, et al. Niche dynamics in space and time. Trends Ecol Evol, 2008, 23: 149-158 


\title{
Dynamic niche: a new foundation for rebuilding theory of community ecology
}

\author{
NIU KeChang ${ }^{1}$, CHU ChengJin ${ }^{2}$ \& WANG ZhiHeng ${ }^{3}$ \\ 1 School of Life Sciences, Nanjing University, Nanjing 210023, China; \\ 2 School of Ecology, Sun Yat-sen University, Guangzhou 510006, China; \\ 3 Institute of Ecology, College of Urban and Environmental Sciences, Peking University, Beijing 100871, China
}

Ecology aims to elucidate how organisms interact with other organisms and their environment. As a fundamental concept of ecological research, the term ecological niche was coined to measure the ecological significance of a species in terms of speciesspecific attributes in relation to the acquisition of environmental resources and impact on the environment. By quantifying species niche, ecologists intended to formulate general rules for controlling various ecological processes, such as species distribution, community assembly, dynamics of the food web structure, and functioning. Over the course of a century, the concept of ecological niche has evolved; however, there is limited information about its essence and meaning. Furthermore, we lack knowledge about its quantification in practice, which means that niche-based theories are faced with significant challenges. Hence, we briefly reviewed the evolutionary history of the niche concept and highlighted its fundamental importance in building the theory of community ecology, such as competition exclusion, species coexistence, and community assembly. From the perspective of feedback dynamics between organisms and the environment, we rethink why the ecological niche can be used to explain various existing patterns but predict nearly nothing. We draw a conclusion that ecological niche not only measures the ecological significance of species that drives community dynamics but also presents it as an emerging consequence of the adaptation of species to the community environment. Moreover, it is essentially a conceptual framework, instead of an analytical approach, for understanding ecological rules. By reconciling the perspectives of reductionism and holism, we propose a conceptual framework for a dynamic niche that considers the importance of change in organism attributes, eco-evolutionary feedback, and stochastic processes on the dynamics of species fitness and community assembly. By updating the classical niche concept into a dynamic one, we aim to establish the foundation for the revision of theories of community ecology.

ecological niche, community assembly, biodiversity, species coexistence, neutral theory

doi: $10.1360 /$ SSV-2021-0160 